\title{
Probing the electronic structure of Ni-Mn-In-Si based Heusler alloys thin films using magneto-optical spectra in martensitic and austenitic phases.
}

\author{
A. Novikov ${ }^{1}$, A. Sokolov ${ }^{2}$, E. A. Gan'shina ${ }^{1}$, Abdiel Quetz ${ }^{3}$, I. S. Dubenko ${ }^{3}$, S. \\ Stadler $^{4}$, N. Ali ${ }^{3}$, I. S. Titov ${ }^{1}$, I. D. Rodionov ${ }^{1}$, E. Lähderanta ${ }^{5}$, A. Zhukov ${ }^{6}$, A. B. \\ Granovsky $^{1}$, R. Sabirianov ${ }^{7}$
}

${ }^{1}$ Department of Physics, Lomonosov Moscow State University, Moscow, 119991 Russia

${ }^{2}$ UNL Department of Physics and Astronomy, Lincoln, NE 68588, USA

${ }^{3}$ Department of Physics, Southern Illinois University, Carbondale, IL 62901 USA

${ }^{4}$ Department of Physics and Astronomy, Louisiana State University, Baton Rouge, Louisiana 70803, USA.

${ }^{5}$ Lappeenranta University of Technology, 53851, Finland

${ }^{6}$ Dpto. de Física de Materiales, Fac. Químicas, UPV/EHU, 20018, San Sebastian, Spain and IKERBASQUE, Basque Foundation for Science, 48011 Bilbao, Spain

${ }^{8}$ Department of Physics, University of Nebraska at Omaha, Omaha, NE 68182, USA

\begin{abstract}
Thin films of $\mathrm{Ni}_{52} \mathrm{Mn}_{35-\mathrm{x}} \mathrm{In}_{11+\mathrm{x}} \mathrm{Si}_{2}$ were fabricated by magnetron sputtering on $\mathrm{MgO}(001)$ single crystal substrates. Magnetization as function of temperature for $\mathrm{Ni}_{52} \mathrm{Mn}_{35} \mathrm{In}_{11} \mathrm{Si}_{2}$ exhibits features consistent with a magnetostructural transition (MST) from an austenitic phase to a martensitic phase, similar to the bulk material. We observed that the martensitic transformation is externally sensitive to small changes in chemical composition and stoichiometry. It has been found that thin films of $\mathrm{Ni}_{52} \mathrm{Mn}_{34-\mathrm{x}} \mathrm{In}_{11+\mathrm{x}} \mathrm{Si}_{2}$ with $\mathrm{x}=0$ and 1 undergo a temperature-induced MST or remain in a stable austenitic phase, respectively. Comparison of magneto-optical transverse Kerr effect spectra obtained at $0.5-4.0 \mathrm{eV}$ in the $35-300 \mathrm{~K}$ temperature interval reveal insignificant differences between the martensitic and austenite phases. We found that the field and temperature dependencies of the transverse Kerr effect are quite different from the magnetization behavior, which is attributed to magnetic inhomogeneity across the films. To elucidate the effects of magnetostructural phase transitions on the electronic properties, we performed density functional calculations of the magneto-optical Kerr effect.
\end{abstract}

\section{Introduction}

Heusler alloys exhibit a wide variety of magnetic, electrical, optical, and mechanical properties that are important for fundamental research and promising for applications [1]. Some exhibit magnetostructural transitions (MST), i.e., the simultaneous transformation of crystal structure and magnetic state that gives rise to a number of intriguing phenomena such 
as giant magnetocaloric effects [2, 3], large magnetoresistance, strong mechanical deformations, magnetic shape memory effects, exchange bias, kinetic arrest, etc.[4-6]. NiMn-In-based Heusler alloys with nearly 15 at. \% concentration of In are particularly attractive because magnetostructural phase transitions occur near room temperature in these systems which can be induced by external magnetic fields $[4,6]$. In the bulk alloys, a hightemperature austenitic phase (AP) with a crystal cell of cubic symmetry transforms to a lowtemperature martensitic phase (MP) with a crystal cell of lower symmetry (with tetragonal distortions). While in the AP phase, a second order phase transition from the paramagnetic $(\mathrm{PM})$ to ferromagnetic (FM) state occurs at a Curie temperature $\left(\mathrm{T}_{\mathrm{C}}\right)$ of about 280-300 K. The onset of the MP emerges with the first order phase transition at $T_{M}=250-280 \mathrm{~K}$, resulting in a low magnetization martensitic state, which in turn changes to a ferromagnetic MP at $\mathrm{T}<\mathrm{T}_{\mathrm{CM}}$. However, antiferromagnetic correlations and inclusions of the AP in the martensitic state exist down to very low temperatures (see $[4,7,8]$ and references therein).

The magnetic ordering in the AP and MP strongly depend on composition [4, 6, 9], and changes smaller than 1\% may completely change the type of long-range magnetic order. The vast majority of results on multifunctional properties of Ni-Mn-In-based Heusler alloys have been observed in bulk polycrystalline samples. However, many applications (see for example in $[7,10])$ require the same properties in thin films. Moreover, thin film geometry can significantly simplify the investigations of physical properties that depend on thickness, such as Hall effects, and surface-sensitive effects such as optical and magneto-optical phenomena. However, the fabrication of high-quality films with reproducible and robust properties of bulk materials remains a technological challenge due to the extreme sensitivity of the properties to slight departures from optimal compositions and the "clamping effect" of substrates [11, 12]. Recently, quaternary Ni-Mn-In-Co [10] and ternary Ni-Mn-In [7, 13] alloy thin films with well pronounced MSTs have been fabricated by DC magnetron sputtering and laser-assisted molecular beam epitaxy methods, respectively. Such progress presents an opportunity to study surface sensitive properties of materials owed to MSTs in thin films, and to obtain better insight into the origins of the MST itself.

It is well known that magneto-optical (MO) spectra are extremely sensitive to minute details of electronic structure and therefore can be used for both structural characterization and for the investigation of the transformation of electronic and magnetic structures at phase transitions $[12,14]$. While it can be a very powerful tool for phase transition studies, to the best of our knowledge MO spectroscopy has not been applied to investigate thin films exhibiting MSTs. 
In this paper, we present experimental results on magnetic and magneto-optical properties for $\mathrm{Ni}_{52} \mathrm{Mn}_{35-\mathrm{x}} \mathrm{In}_{11+\mathrm{x}} \mathrm{Si}_{2}$ Heusler alloys thin films of different compositions: $\mathrm{x}=0$ and $\mathrm{x}=1$ which undergo a temperature-induced magnetostructural transition and remain in a stable austenitic phase, respectively. We focused our study on quaternary Ni-Mn-In-Si Heusler alloys due to its sharp dependence of the MCE properties with silicon concentration and to perform magneto-optic studies using austenitic and martensitic phases of the films in the wide temperature interval. [15]. We performed a comparative analysis of their properties, supported by density functional theory calculations of the magneto-optical Kerr effect and site-resolved density of states in the austenite and martensitic phases.

\section{Experimental procedures}

\section{A. Preparation and measurements}

Thin films samples $\mathrm{Ni}_{52} \mathrm{Mn}_{35-\mathrm{x}} \mathrm{In}_{11+\mathrm{x}} \mathrm{Si}_{2}$ samples were fabricated by the simultaneous magnetron sputtering from pure element (3N) targets on MgO (001) substrates. The sample compositions were controlled through careful calibration of the deposition rates for each sputtering gun. During the film deposition, the substrate temperature was held at $320^{\circ} \mathrm{C}$. To improve wettability, a $2 \mathrm{~nm}$ seed layer of pure Ni was deposited prior to film synthesis. The thicknesses of all of the films were $\sim 75 \mathrm{~nm}$. After deposition all samples were annealed in vacuum at $650^{\circ} \mathrm{C}$ for one hour and then rapidly quenched. We used a small resistive heater element which surrounds the substrate without direct contact, and the temperature was measured with a pyrometer. Resulting compositions of the samples were determined by energy-dispersive X-ray spectroscopy (EDX). The presence of a MP or AP in the samples was determined through X-ray diffraction (XRD). Although we maintained the same deposition conditions for all samples, inevitable target aging resulted in some composition deviation, especially in the indium content. Two samples with slightly different compositions due to the $\mathrm{Mn} / \mathrm{In}$ ratio deviation were chosen for further extensive analysis: $\mathrm{Ni}_{52} \mathrm{Mn}_{34} \mathrm{In}_{12} \mathrm{Si}_{2}$ $(\mathrm{x}=1)$ and $\mathrm{Ni}_{52} \mathrm{Mn}_{35} \mathrm{In}_{11} \mathrm{Si}_{2}(\mathrm{x}=0)$, which remains in AP at all temperatures and shows a temperature-induced MST, respectively. Note that a small excess in the relative Ni content was attributed to the presence of the Ni seed layer.

The magnetic properties were measured in the temperature interval from 5-400 K and in magnetic fields up to $50 \mathrm{kOe}$ using a Quantum Design superconducting quantum interference device (SQUID) magnetometer, and also with a vibrating sample magnetometer (Lake Shore VSM) in magnetic fields up to $16 \mathrm{kOe}$. The measurements with the SQUID were performed by an applying the magnetic field perpendicular to the thin film plane. MO 
measurements were carried out in the transverse Kerr effect (TKE) geometry. The sensitivity of our measurements was estimated to be $\approx 10^{-5}$ [16]. An alternating magnetic field was aligned parallel to the plane of thin films and perpendicular to the light plane of incidence.

The spectral, field, and temperature dependencies of the TKE were performed in the energy range of incident light from 0.5 to $4.0 \mathrm{eV}$ in magnetic fields up to $2.7 \mathrm{kOe}$, and in the temperature range from 35 to $300 \mathrm{~K}$. The temperature dependencies of magnetization, $\mathrm{M}(\mathrm{T})$, and the TKE parameter $\delta(\mathrm{T})$ MO response, were determined during heating after the samples were cooled from $300 \mathrm{~K}$ to $35 \mathrm{~K}$ in zero magnetic field (ZFC) and during a field-cooling cycle (FCC).

\section{B. Computational details}

In order to elucidate the effects of the phase transitions on the TKE, we performed time-dependent density functional theory (DFT) calculations using a FP-LAPW method [17]. A local density approximation (LDA) with the Perdew-Wang exchange-correlation functional in the parameterization of Ceperley-Alder was applied [18]. In order to analyze the effects of the magnetic phase transformation and quantify the significance of the relative $\mathrm{Mn} / \mathrm{In}$ concentration on the electronic structure and TKE behavior, we considered $\mathrm{Ni}_{8} \mathrm{Mn}_{4+\mathrm{x}} \mathrm{In}_{4-\mathrm{x}}$ $(\mathrm{x}=0,1,2)$ in ferromagnetic (FM) cubic and ferrimagnetic (FiM) tetragonal unit cells, i.e., 16 atoms in the unit cell. Lattice parameters were taken from the experimental data as $a=5.5785$ $\AA$. We assumed the Ni atoms occupied the Wyckoff positions at $(0,0,0)$ and $\mathrm{Mn}$ and In atoms occupied positions at $(1 / 4,1 / 4,1 / 4)$ in the cubic lattice. Mn sites in $\mathrm{Ni}_{2} \mathrm{MnIn}$ were coordinated by nearest neighbors of $\mathrm{Ni}$ and In. The Mn-Mn interatomic distance is large and the exchange coupling is ferromagnetic. Mn sites occupying In positions created shorter MnMn bonds that exhibited antiferromagnetic coupling. A $12 \times 12 \times 12 \mathrm{k}$-grid mesh was used to sample the Brillouin zone in the cubic lattice, and $8 \times 6 \times 4$ in the tetragonal distorted lattice.

The diagonal and off-diagonal matrix elements of the optical conductivity tensor were calculated using linear-response time-dependent density functional theory (TDDFT) [17]. The transverse magneto-optical Kerr effect constitutes a variation of the reflected light intensity due to the magnetization of a ferromagnetic sample and can be described as:

$$
I_{P} \sim r_{p p}\left(1+2 \mathfrak{R}\left(\frac{r_{p p}(m a g)}{r_{p p}}\right) m\right)
$$

where $\mathrm{m}$ is the magnetization, $\mathrm{r}_{\mathrm{pp}}$ and $\mathrm{r}_{\mathrm{pp}}$ (mag) express the part of reflection coefficient that does independent of and linear with the $\mathrm{m}_{\mathrm{x}}$ magnetization component, respectively [19]. For p-polarized waves the TKE spectra effects can be written as: 


$$
\delta_{p}=2 \sin (2 \varphi) \frac{A_{1}}{A_{1}^{2}+B_{1}^{3}} \varepsilon_{x y}^{\prime}+2 \sin (2 \varphi) \frac{B_{1}}{A_{1}^{2}+B_{1}^{3}} \varepsilon_{x y}^{\prime \prime}
$$

where $\varepsilon_{x y}^{\prime}$ and $\varepsilon_{x y}^{\prime \prime}$ are the off-diagonal components of the dielectric permittivity tensor, while A and B depend on the diagonal component of permittivity tensor as follows:

$$
A_{1}=\varepsilon_{0}^{\prime \prime}\left(2 \varepsilon_{0}^{\prime} \cos (\varphi)^{2}-1\right), B_{1}=\left(\varepsilon_{0}^{\prime 2}-\varepsilon_{0}^{\prime 2}\right) \cos (\varphi)^{2}+\varepsilon_{0}^{\prime}-\sin (\varphi)^{2},
$$

where $\varphi$ is incidence angle of the light measured from the normal of the sample surface. Since the components of the tensor in visible and near ultra-violet spectra are defined by interband transitions, i.e., the density of states of valence and occupied states (together with matrix elements of momentum operator) [17], a measurement of the MO spectra provides valuable information about the distribution of electronic states of the Heusler alloys in a wide range of energies and its transformation upon a magnetostructural transition.

\section{RESULTS AND DISCUSSIONS}

\section{A. Structural and magnetic properties.}

The XRD data are shown in Figure 1. While all of the peaks in the $\mathrm{Ni}_{53} \mathrm{Mn}_{33} \mathrm{In}_{12} \mathrm{Si}_{2}$ $(\mathrm{x}=1)$ scans can be ascribed to the austenite cubic phase, an additional peak occurs in $\mathrm{Ni}_{52} \mathrm{Mn}_{35} \mathrm{In}_{11} \mathrm{Si}_{2}(\mathrm{x}=0)$ at $2 \Theta=46.4^{\circ}$, which can be attributed to the presence of the tetragonal phase at ambient temperature.

Figure 2 shows the temperature dependencies of the ZFC and FC magnetization of the samples obtained at $\mathrm{H}=250$ Oe with the SQUID. Both samples demonstrate a sharp increase in $\mathrm{M}(\mathrm{T})$ below $300 \mathrm{~K}$ with cooling. Negligible temperature hysteresis with a width within the experimental error clearly indicates a second-order phase transition from a paramagnetic AP to a ferromagnetic AP. The Curie temperature, determined by the slope of $\mathrm{M}(\mathrm{T})$, was found to be $\approx 275 \mathrm{~K}$ and $285 \mathrm{~K}$ for samples with $\mathrm{x}=0$ and 1, respectively. Such a noticeable sensitivity of Tc (about $10 \mathrm{~K}$ ) on slight changes in In-concentration has also been reported for the bulk $N i_{50} M n_{50-x} I n_{x}$ system (see for example [8] and [20]). Further cooling reveals a dramatic difference in magnetic behavior of the samples. The magnetization of the field cooled (FC) ( $\mathrm{H}=250$ Oe) $\mathrm{Ni}_{52} \mathrm{Mn}_{34} \mathrm{In}_{12} \mathrm{Si}_{2}$ (Fig. 2a) demonstrates no additional phase transitions, but does show a broad maximum at $140 \mathrm{~K}$ that weakens with further cooling. When the temperature of the sample is lowered in the absence of external magnetic field (ZFC), the observed magnetization decline is much more pronounced, and maximum of magnetization curve occurs at $240 \mathrm{~K}$, which can be explained by the effect of magnetic anisotropy of the sample. In contrast, the $\mathrm{M}(\mathrm{T})$ of $\mathrm{Ni}_{52} \mathrm{Mn}_{35} \mathrm{In}_{11} \mathrm{Si}_{2}$ exhibits a clear hysteretic 
behavior at 175-250 K (Fig. 2b), which is evidence of a first order phase transition, similar to the MST observed in the bulk materials. In contrast to the bulk, the transition was not accompanied by a sharp change in the magnetization, and the hysteresis occurs in a wider temperature range. A strong exchange bias observed at $5 \mathrm{~K}$ (see inset of Fig. 2b compared to the inset of Fig. 2a) suggests the presence of strong antiferromagnetic correlations in the sample with $\mathrm{x}=0$. Hence, summarizing the magnetic and $\mathrm{XRD}$ data, it is conceivable to conclude that, while the sample with $\mathrm{x}=1$ remains in the austenite cubic phase in the entire temperature range, both the crystalline and magnetic structures of sample with $x=0$ are not homogenous below $250 \mathrm{~K}$ and contains a mixture of MP and AP states. Such inhomogeneity emphasizes the importance of the interfacial effects in thin films. It has been shown by several research groups that the clamping effect of substrates on adjacent layers prevents the latter from transforming to the martensitic phase [11, 12 and ref. therein]. However, our experimental data cannot rule out the scenario where the MST proceeds directly from a ferromagnetic austenitic to ferromagnetic martensitic phase without the phase with low magnetization.

\section{B. Magneto-optical analysis}

In order to probe the effects of the structural transitions on the electronic structure, we have performed a MO study of these samples. We note that the characteristic probing depth of MO methods is $\lambda / 4 \pi \mathrm{k}$ (where $\mathrm{k}$ is the coefficient of absorption), which is about $20-30 \mathrm{~nm}$ in the visible range and comparable with the film thickness. [22]). Our MO measurements (Fig. 3) were found to be in good agreement with the magnetic data. Indeed, the hysteretic behavior of the temperature dependence of the MO response was observed for sample with $\mathrm{x}=0$ and spans from $280 \mathrm{~K}$ down to $150 \mathrm{~K}$, and is not observed for sample with $\mathrm{x}=1$ (compare with Fig. 2 a, and b). For temperatures below $150 \mathrm{~K}$, the MO response in the FC regime decreases with temperature, while the magnetization, measured at the same field, increases. This can be understood in the case where the magnetic anisotropy of the surface layers, which are primarily responsible for the MO response, is larger than that of the layers close to the substrate. This observation supports our earlier conclusions made from the magnetic and XRD data. Similar results were previously observed in studies of other Heusler alloys (e.g., $\mathrm{Ni}_{2} \mathrm{MnGa}$ [23]). The difference in magnetic anisotropy might be caused by magnetic inhomogeneity across the film thickness.

From Figs. 3 we can also see that the TKE for $\mathrm{Ni}_{52} \mathrm{Mn}_{34} \mathrm{In}_{12} \mathrm{Si}_{2}$ in the AP is much larger than that for sample $\mathrm{Ni}_{52} \mathrm{Mn}_{35} \mathrm{In}_{11} \mathrm{Si}_{2}$. Since the TKE signal depends linearly on the 
magnetic moment at the corresponding field, this confirms the presence of antiferromagnetic short-range order in the sample with $\mathrm{x}=0$.

Previous MO investigations of bulk Ni-Mn-In samples [24, 25] demonstrated that the MO spectra for the AP and MP are very similar. Here we found that this is also true for thin films. Figure 4 demonstrates MO spectra of samples in martensitic and austenite phases and, indeed, the overall profile and spectral positions of positive and negative peaks in the MO spectra for the sample with $x=1$ (which is in the AP) and the sample with $x=0$ (which is mostly in the MP below $150 \mathrm{~K}$ ) are very similar (Fig. 4). As mentioned above, the amplitudes of the MO responses for these samples are quite different due to antiferromagnetic correlations in sample with $\mathrm{x}=0$, but the shape of the MO spectra profiles are very similar. Small differences in the spectra below $1.5 \mathrm{eV}$ may be attributed to the difference in compositions of samples.

At low temperatures, the TKE of $\mathrm{Ni}_{52} \mathrm{Mn}_{35} \mathrm{In}_{11} \mathrm{Si}_{2}$ is almost constant after the magnetization is saturated at fields $\mathrm{H} \geq 2.7 \mathrm{kOe}$. It is interesting to note that the TKE observed in $\mathrm{Ni}_{52} \mathrm{Mn}_{35} \mathrm{In}_{11} \mathrm{Si}_{2}$ is virtually identical for $\mathrm{T}=35 \mathrm{~K}$ and $140 \mathrm{~K}$ (Fig. 4). This behavior can be related to the co-existence of AP and MP in the wide temperature interval with different magnetic structures [29].

The similarity of the TKE spectra in the austenite and martensitic phases is interesting since it is believed (see for example [26]) that the MST is accompanied by noticeable changes in electronic structure, particularly, a large difference in the density of states at the Fermi level for the MP and AP. This is supported by a large change in resistivity of the bulk systems at the MST. However, recent experiments on the Hall effect [8], specific heat [27], and thermopower [28] form a more complicated view of the changes in electronic structure. While these experiments give insight into the electronic structure at the Fermi level, the MO transitions occur between occupied and unoccupied electronic states. Thus, the shapes of the TKE spectra depend on the distribution of states across the wide energy range of occupied and unoccupied states (as well as on the dependence of momentum matrix elements). Therefore, keeping in mind that the MO experiments for thin films are more reliable than for bulk samples due to the high-quality of the surfaces, our experiments support the hypothesis of very limited modification in the electronic structure for the studied Ni-Mn-In-based Heusler alloys between samples with or without a MST.

\section{Numerical results for Transverse Kerr Effect}


In order to understand the sensitivity of the TKE on small composition and chemical order variations, we calculated the dielectric tensor and optical conductivity in $\mathrm{Ni}_{8} \mathrm{Mn}_{4+\mathrm{x}} \mathrm{In}_{4-\mathrm{x}}$ using time-dependent density functional theory (DFT) calculations implemented in the FPLAPW method. We focused our attention on the relative Mn/In content in the interval from $x=0$ to 2. In accordance with previously reported results, where competition between ferromagnetic and antiferromagnetic interactions has been theoretically determined for NiMn-based Heusler compounds with the extra $\mathrm{Mn}[29,30], \mathrm{Ni}_{8} \mathrm{Mn}_{4} \mathrm{In}_{4}$ forms a perfect cubic Heusler alloy and Mn sites are separated by a large distance ( $4 \AA)$. We found that the exchange interactions between the Mn sites are ferromagnetic in this structure and the cubic lattice has a lower energy compared to the tetragonal one. At $\mathrm{x} \neq 0, \mathrm{Mn}$ atoms partially occupy In sites, increasing the degree of chemical disorder and shortening the distance between $\mathrm{Mn}$ at the substitution sites from the nearest Mn neighbors to $~ 2.9 \AA$. Our calculations confirm that there is a structural instability in this case. While the FM cubic state is locally stable, the ground state of the system shows significant structural distortions, where the Mn atoms in In positions shift towards one of the original Mn sites in the local environment as shown in Figure 5a. As a result of the reduced Mn-Mn distances the exchange interactions become antiferromagnetic, causing ferrimagnetic ordering and a reduced magnetization, which is supported by the direct calculations of exchange interactions in NiMnIn systems using density functional theory calculations [31, 32] as well as Mossbauer measurements in Fe doped systems [33]. However, the local magnetic moment on the $\mathrm{Mn}$ sites is nearly unchanged in the FM and FiM configurations. Thus, Mn-Mn pairs with the short interatomic distances do not contribute to the magnetization, and the overall value of the magnetization reduces. In contrast, the Ni sites remain unchanged and have magnetic states that are similar in both the FM and FiM states. Furthermore, due to the heredity of the FM AP and the FiM MP states, there is also a heredity in electronic structures of these states. Figure $5 b$ show the DOS of the FM and FiM states of the $\mathrm{Ni}_{8} \mathrm{Mn}_{5} \mathrm{In}_{3}$ unit cell. The transformation from the FM to FiM states causes a slight change in the shape of the peaks in the DOS. Particularly, the Mn atoms that occupy In positions experience a narrowing and shift of the DOS peaks. However, the overall shapes of partial the site-projected DOS does not change significantly, in accordance with our experimental observation.

The calculated TKE spectra presented in Figure 6 show that there is overall agreement between the TKE of the calculated and experimentally analyzed samples. The discrepancy between calculated results and experimentally observed data below $1 \mathrm{eV}$ is most likely due to the fact that our first principles calculations consider only inter-band transition contributions 
to the TKE. While at lower energies intra-band magneto-optical transitions become more important, above $2 \mathrm{eV}$ they do not play any role. Quantitative analysis of intra-band transitions in thin films is very difficult and out of the scope of this paper A simple, classical phenomenological Drude model is typically used to analyze the MOKE or TKE data. However, results are very sensitive to the calculated plasma frequency and Lorenzian broadening.

Since our sample in the martensitic phase contains a fraction of austenite phase (see discussion above) a direct comparison is probably not worthwhile. We have calculated the electronic structures of NiMnIn alloys in the tetragonal unit cell for three compositions of Mn to model the effect of composition, as well as antiferromagnetic correlations in the system. The TKE spectra for ferromagnetic systems of $\mathrm{Ni}_{8} \mathrm{Mn}_{4+\mathrm{x}} \mathrm{In}_{4-\mathrm{x}}$ show the largest (negative) peaks at $2.7 \mathrm{eV}$ and $3.8 \mathrm{eV}$. However, the relative strength of these peaks change with concentration. The peak at $2.7 \mathrm{eV}$ is more prominent at $\mathrm{x}=0$, i.e., at lower $\mathrm{Mn}$ concentration, while the peak at $3.8 \mathrm{eV}$ becomes dominant at larger Mn content. The Mn concentration in experimentally produced films is closest to $\mathrm{Ni}_{8} \mathrm{Mn}_{5} \mathrm{In}_{3}$ (i.e. for $\mathrm{x}=1$ ).

It can be seen that the presence of antiferromagnetically arranged Mn sites in these compounds modifies the TKE signals. The magnitude of the TKE signal decreases due to the reduction in magnetization upon reversal of the local spin moments of the Mn sites. The larger number of antiferromagnetically correlated spins, the larger the reduction of the signal. For example, the reduction of magnetization in $\mathrm{Ni}_{8} \mathrm{Mn}_{6} \mathrm{In}_{2}$ is $\sim 67 \%$ (from $22 \mu_{\mathrm{B}}$ to $7 \mu_{\mathrm{B}}$ ) upon reversal of Mn magnetic moments in the In positions, trailed by the reduction of the TKE signal by the same factor. Similarly, the magnetization in $\mathrm{Ni}_{8} \mathrm{Mn}_{5} \mathrm{In}_{3}$ reduces by $38 \%$ in the ferrimagnetic arrangement, and the TKE decreases by 30\%. The experimental spectra for the films have a maximum in the magnitude of the TKE at $-2.6 \mathrm{eV}$ and it decreases considerably between the FM films with larger magnetization and films with lower magnetization due to antiferromagnetic correlations.

Besides this expected reduction in the signal magnitude, there is also an increase in the relative strength of the peak at $2.7 \mathrm{eV}$ compared to the one near $3.8 \mathrm{eV}$. We find that the calculated TKE peak at $\sim 2.7 \mathrm{eV}$ is largest both in the ferromagnetic cubic phase at lower Mn content and in the ferrimagnetic systems. The experimental spectra show little variation in the shape of the TKE spectra between the two films due to the similarity of two spectra and, partly, due to the substantial mix of AP ferromagnetic phases in these films.

\section{Conclusions}


Magnetron sputtering can be used to fabricate Ni-Mn-In-based Heusler alloy thin films that exhibit martensitic transitions. The magnetic properties of the samples are highly sensitive to composition and the details of fabrication. Evidence of martensitic transitions in the magnetothermal behavior and magneto-optical response are quite different, indicating thin film inhomogeneity in the thickness. Transverse magneto-optical spectra in the austenitic and martensitic phases are similar, which is evidence of a hereditary connection between the two phases and the similarity between their electronic structures. Density functional calculations show limited differences in the densities of states and TKE of NiMnIn alloys in the ferromagnetic states and state with a ferrimagnetic component.

\section{Acknowledgements}

This work was supported by the National Science Foundation (NSF) through NSF DMR1310542 (A.S.), the Office of Basic Energy Sciences, Material Science Division of the U.S. Department of Energy, DOE Grant No. DE-FG02-06ER46291 (SIU), and DE-FG0213ER46946 (LSU), and by the Russian Foundation for Basic Research (№ 15-02-01976: MSU and No. 16-32-00460 -mol-a ?). 


\section{FIGURES}

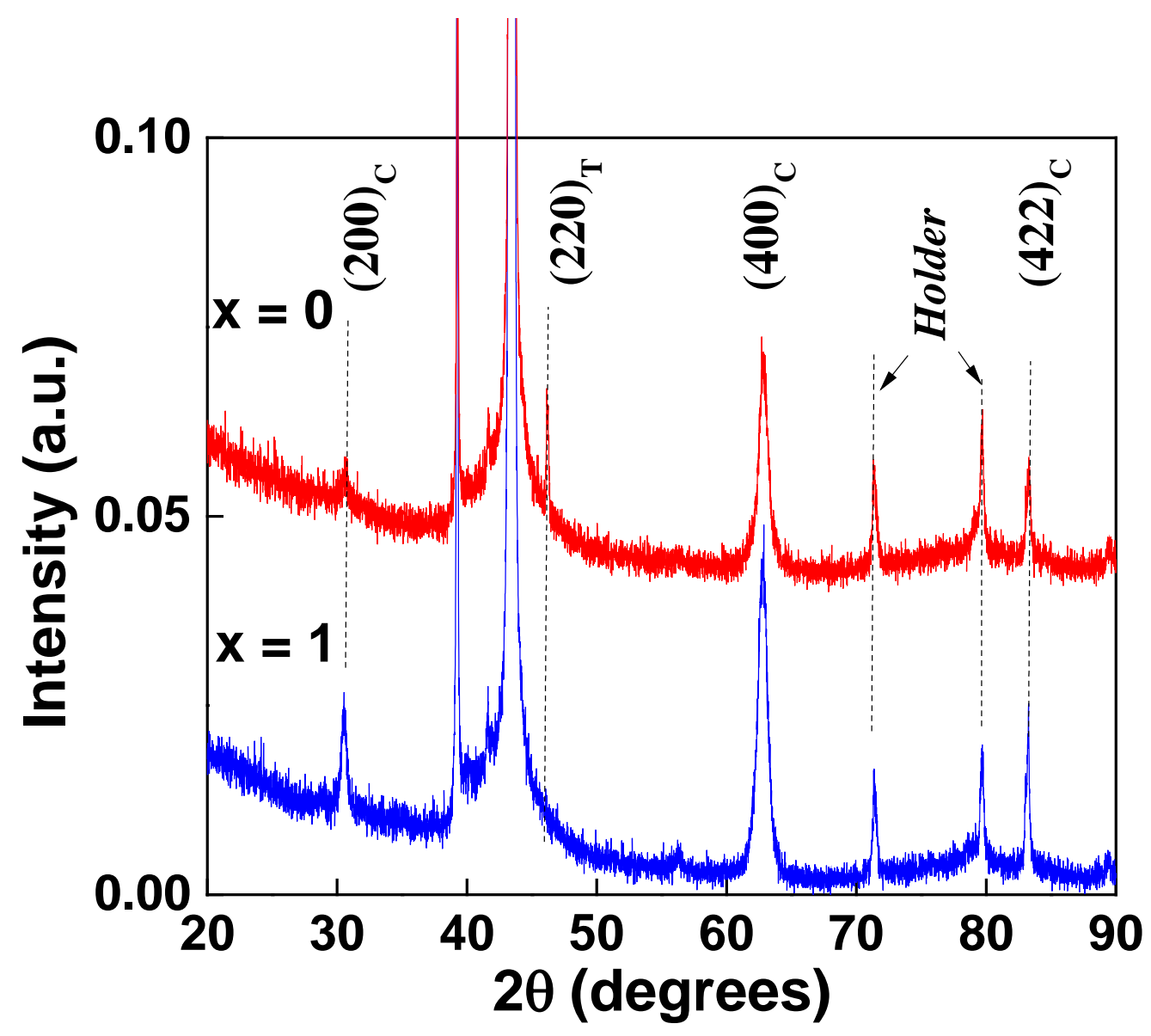

Figure 1. Room temperature XRD patterns for $\mathrm{Ni}_{52} \mathrm{Mn}_{35-\mathrm{x}} \mathrm{In}_{11+\mathrm{x}} \mathrm{Si}_{2}$ thin films with $\mathrm{x}=0$ (upper curve) and $\mathrm{x}=1$ (lower curve). Labeled peaks correspond to the cubic austenitic phase (C) and the tetragonal phase (T). 

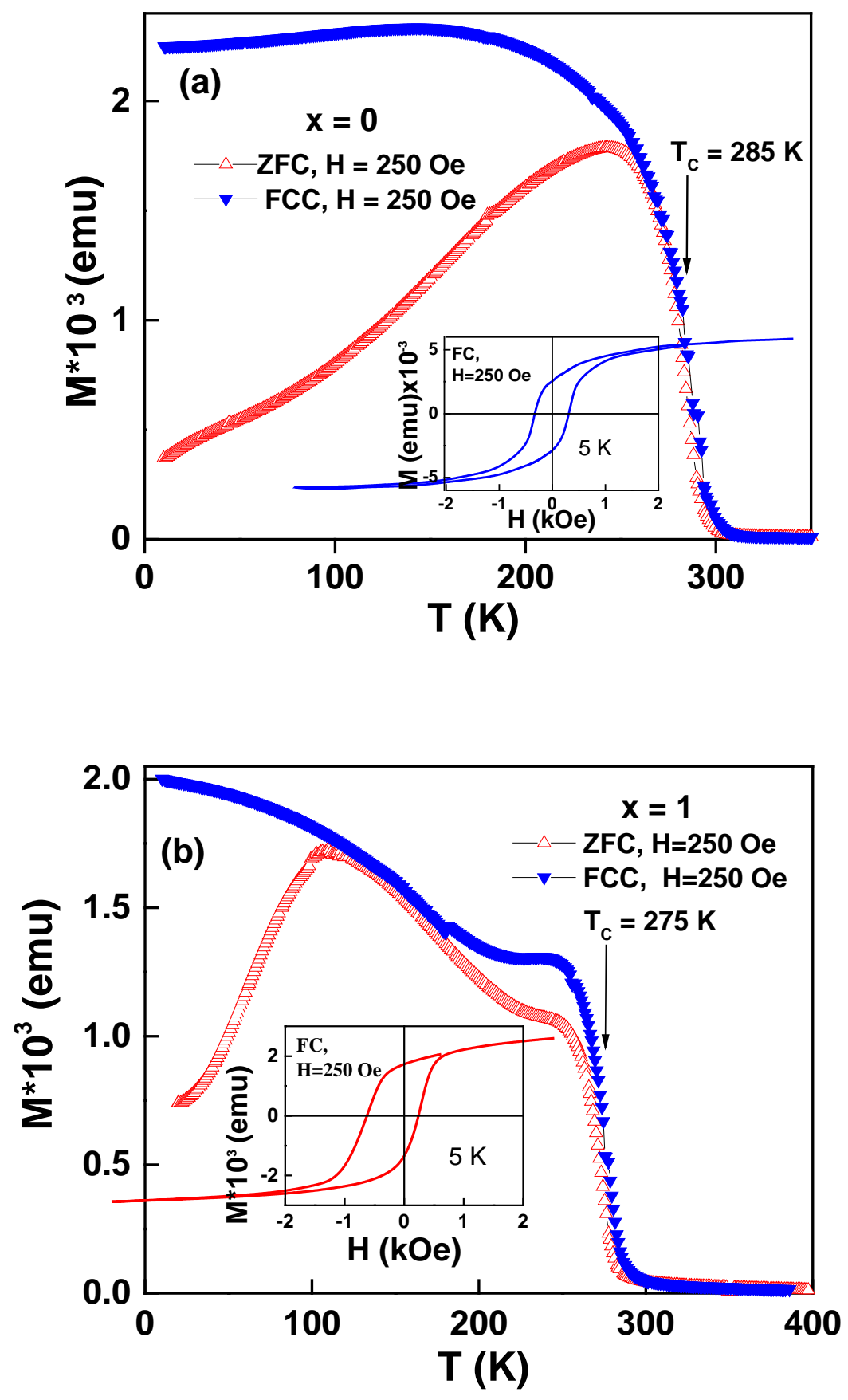

Figure 2. Temperature dependent magnetization $M(T)$ for $\mathrm{Ni}_{52} \mathrm{Mn}_{35-\mathrm{x}} \mathrm{In}_{11+\mathrm{x}} \mathrm{Si}_{2}$ thin films with $\mathrm{x}=1$ (a) and $\mathrm{x}=0$ (b). The inset shows $\mathrm{M}(\mathrm{H})$ curves for the samples and indicates the presence of exchange bias in the sample with $\mathrm{x}=0$. 


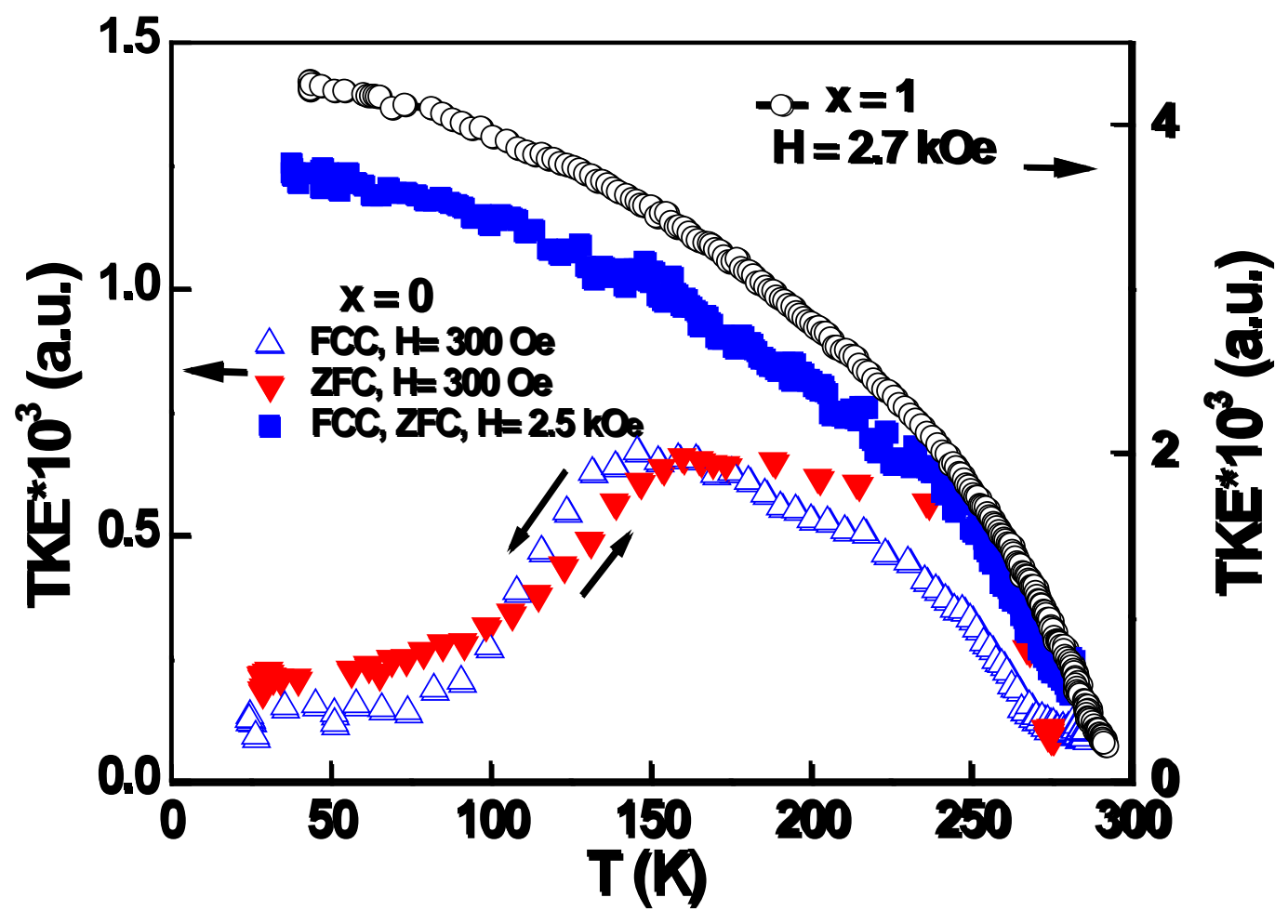

Figure 3. The temperature dependence of the TKE for the $\mathrm{Ni}_{52} \mathrm{Mn}_{35-\mathrm{x}} \mathrm{In}_{11+\mathrm{x}} \mathrm{Si}_{2}$ samples with $x=1$ (a), which remains in the austenitic phase in a magnetic field of $2.7 \mathrm{kOe}$, and for the sample with $\mathrm{x}=0$ (b) in magnetic fields of $300 \mathrm{Oe}$ (in the ZFC and FC regimes) and $2.5 \mathrm{kOe}$ (in the FH regime) (see details in the text). 


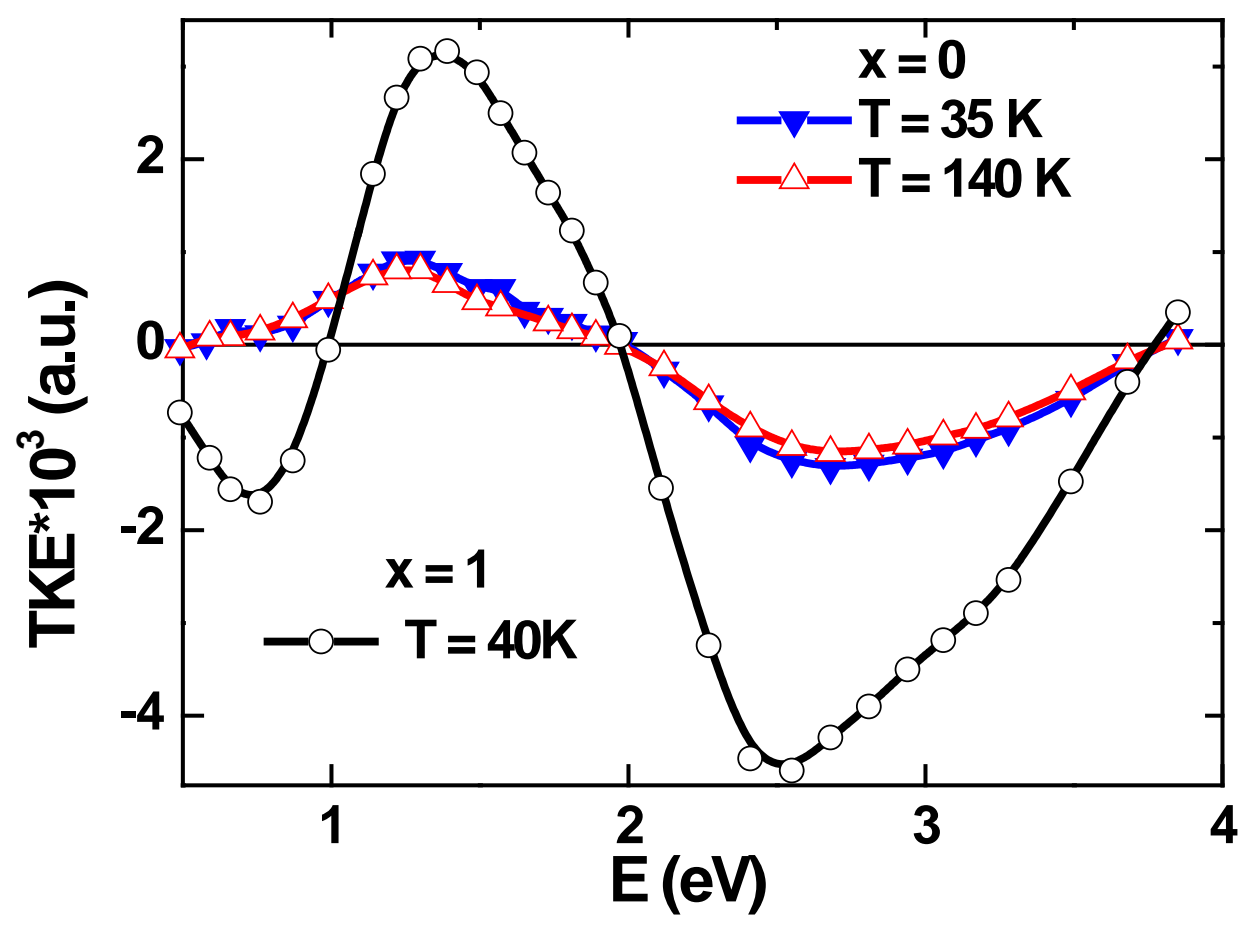

Figure 4. Spectral dependence of the TKE for $\mathrm{Ni}_{52} \mathrm{Mn}_{35-\mathrm{x}} \mathrm{In}_{11+\mathrm{x}} \mathrm{Si}_{2}$ samples with $\mathrm{x}=1$ (austenitic phase) and for a sample with $\mathrm{x}=0$ (austenitic and martensitic phases). 


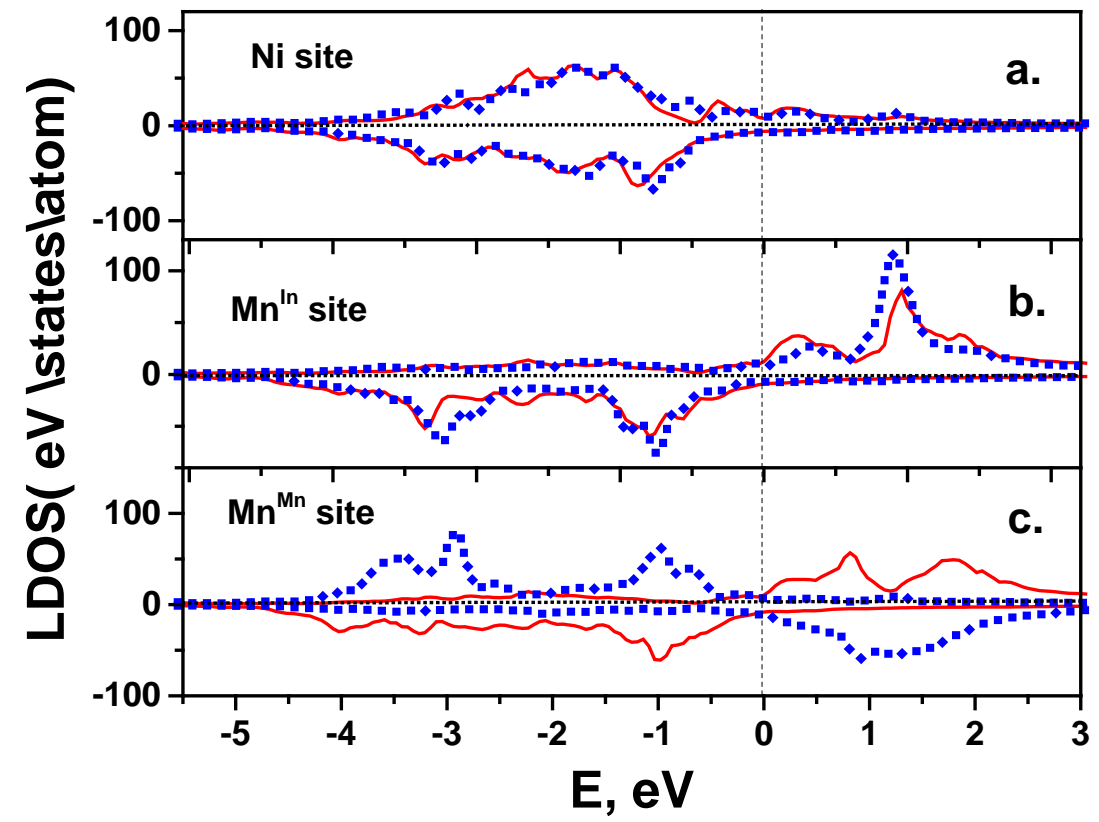

Figure 5. Partial (site projected) DOS of $\mathrm{Ni}_{8} \mathrm{Mn}_{5} \mathrm{In}_{3}$ in FM (solid red) and FiM (dashed blue) states for: (a) the Ni site, (b) the Mn substituted for In $\left(\mathrm{Mn}^{\mathrm{In}}\right)$, and (c) the $\mathrm{Mn}$ in regular $\mathrm{Mn}$ site $\left(\mathrm{Mn}^{\mathrm{Mn}}\right)$. 


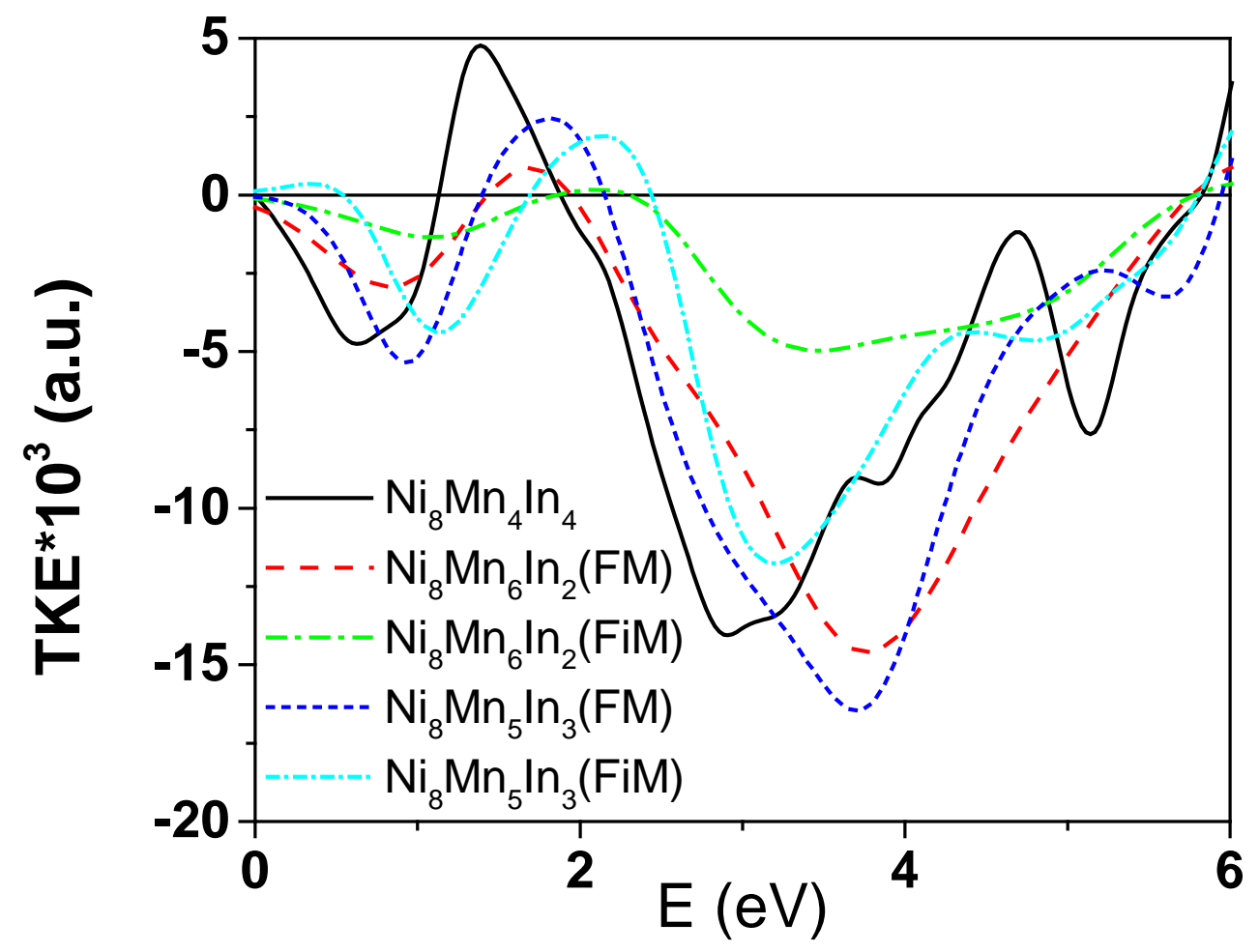

Figure 6. TKE spectra calculated using TDDFT for supercells of $\mathrm{Ni}_{8} \mathrm{Mn}_{4+\mathrm{x}} \mathrm{In}_{4-\mathrm{x}}$ for three concentrations, $\mathrm{x}=0,1,2$. 


\section{References}

[1] T. Graf, S. Parkin, C. Felser, IEEE Trans. Magn. 47, 367 ( 2011).

[2] V. K. Sharma, M K Chattopadhyay, R. Kumar, T. Ganguli, P. Tiwari and S. B. Roy J. Phys.: Condens. Matter, 19 (49), 496207, (2007)

[3] V K Sharma, M K Chattopadhyay and S B Roy, J. Phys. D: Appl. Phys, 40, (7), 1869 (2007)

[4] W. Ito, Y. Imano, R. Kainuma, Y. Sutou, K. Oikawa, K. Ishida, Metal. Mater Trans. A, 38 (4), 759 (2007)

[5] I. Dubenko, T. Samanta, A. K. Pathak, A. Kazakov, V. Prudnikov, S. Stadler, A.

Granovsky, A. Zhukov, N. Ali, J.Magn.Mag.Mater.324, 3530 (2012).

[6] I. Dubenko, A. Quetz, S. Pandey, A. Aryal, M. Eubank, I. Rodionov, V. Prudnikov, A.

Granovsky, E. Lahderanta, T. Samanta, A. Saleheen, S. Stadler, N. Ali, J.Magn.Mag.Mater. 383, 186 (2015).

[7] A. Sokolov, Le Zhang, Dubenko, T. Samanta, S. Stadler, and N. Ali, Appl. Phys. Lett. 102, 072407 (2013).

[8] A. K. Pathak, G. Bhoj, M. Khan, I. Dubenko, S. Stadler, and N. Ali, J. Appl. Phys. 103, $07 F 315$ (1-3) (2008), and A. B. Granovskii, V. N. Prudnikov, A. P. Kazakov, A. Zhukov, I. Dubenko, JETP 115, 805 (2012).

[9] Y. J. Huang, Q. D. Hu, and J. G. Li Design in Ni-Mn-In magnetic shape-memory alloy using compositional Maps, Appl. Phys. Lett. 101, 222403 (2012)

[10] R. Niemann, O. Heczko, L. Schultz, and S. F€ahler, Appl. Phys. Lett. 97, 222507 (2010).

[11] S. Granovsky, I. Gaidukova, A. Sokolov, A. Devishvili, V. Snegirev Solid State Phenomena 233, 666 (2015)

[12] A. Sokolov, E. Kirianov, A. Zlenko, A. Quetz, A. Aryal, S. Pandey, I. Dubenko, S.

Stadler, N. Ali, N. Al-Aqtash, R. Sabirianov, AIP Advances, 6, 056211 (2016)

[13] M. V. Lyange, M. V. Gorshenkov, A.V. Bogach, M. Ohtsuka, H. Miki, T. Takagi, V. V. Khovaylo, "Structural and Transport Properties of Ni45Mn40In15 Thin Films", Solid State Phenomena, 233-234, 670 (2015)

[14] G. Y. Guo and H. Ebert Phys. Rev. B 50, 10377(R) (1994)

[15] A. K. Pathak, I. Dubenko, S. Stadler, and N. Ali, J. Phys D: Appl. Phys. (Fast track comm.) 41, 202004 (2008)

[16] E.A. Balykina, E.A. Gan’shina, G.S. Krinchik, Sov. Phys. JETP, 66,1073 (1987) 
[17] A. Gulans, S. Kontur, C. Meisenbichler, D. Nabok, P. Pavone, S. Rigamonti, S.

Sagmeister, U. Werner and C. Draxl, J. Phys.: Condens. Matter 26363202 (2014)

[18] J. P. Perdew and Y. Wang Phys. Rev. B 45, 13244 (1992)

[19] A. K. Zvezdin and V. A. Kotov, Modern Magneto-Optics and Magneto-Optical Materials (IOP Publishing, Bristol, 1997)

[20] T. Krenke, M. Acet, E. F. Wassermann, X. Moya, L. Mañosa, and A. Planes Phys. Rev.

B 73, 174413 (2006)

[21] B. Hernando , J.L. Sa'nchez Llamazares , J.D. Santos, M.L. Sa'nchez , L. Escoda , J.J.

Suñol, R. Varga , C. Garc1', J. Gonzalez, J. Magn. Magn Mater. 321763 (2009).

[22] G. Traegert, L. Wenzel, A. Hubert, Phys. Stat. Sol.(a), 131, 201 (1992)

[23] A. Novikov, E. Gan'shina, A. Granovsky, A. Zhukov and V. Chernenko, Solid Sate Phenomena, 190, 335 (2012).

[24] E. Gan’shina, A. Novikov, V. Chernenko, J. Barandiaran, E. Cesari, I. Rodionov, I. Titov, V. Prudnikov, A. Granovsky, Solid State Phenomena, 233-234 , 225 (2015).

[25] A. Novikov, E. Gan’shina, L. Gonzalez-Legarreta, V.M. Prida, B. Hernando, A. Granovsky, Solid State Phenomena 233-234, 200 (2015).

[26] P. Entel, V.D. Buchelnikov, V.V. Khovailo, A.T. Zayak, W.A. Adeagbo, M.E. Gruner, H.C. Herper, E.F. Wassermann, J. Phys. D: Appl.Phys. 39, 865 (2006).

[27] T. Kihara, X. Xu, W. Ito, R. Kainuma, and M. Tokunaga, Phys. Rev. B 90, 214409 (2014).

[28] I. Dubenko, A. Granovsky, E. Lahderanta, M. Kashirin, V. Makagonov, A. Aryal, A. Quetz, S. Pandey, T. Samanta, S. Stadler, D. Mazumdar, N.Ali, J.Magn. Mag. Mater. 401, 1145 (2016).

[29] D. Comtesse, M. E. Gruner, M. Ogura, V. V. Sokolovskiy, V. D. Buchelnikov, A. Grünebohm, R. Arróyave, N. Singh, T. Gottschall, O. Gutfleisch, V. A. Chernenko, F. Albertini, S. Fähler, and P. Entel, Phys. Rev. B 89, 184403 (2014).

[30] S. Aksoy, M. Acet, T. Krenke, E. F. Wassermann, M. Gruner, P. Entel, L. Manosa, A. Planes and P. P. Deen:, Springer Series in Materials Science 148, 67 (2012)

[31] V. D. Buchelnikov, P. Entel, S. V. Taskaev, V. V. Sokolovskiy, A. Hucht, M. Ogura, H. Akai, M. E. Gruner, and S. K. Nayak, Phys. Rev. B 78, 184427 (2008).

[32] C. L. Tan, Y. W. Huang, X. H. Tian, J. X. Jiang, and W. Cai, Origin of magnetic properties and martensitic transformation of Ni-Mn-In magnetic shape memory alloys Appl. Phys. Lett. 100, 132402 (2012). 
[33] Khovaylo VV, Kanomata T, Tanaka T, Nakashima M, Amako Y, Kainuma R, Umetsu RY, Morito H, Miki H, Phys. Rev. B 80, 144409 (2009). 